significantly better (71.4 vs $43.6 \%$, OR: 3.2 [95\% CI: 1.4 to 7.6] for sustained ROSC; 57.1 vs $25.9 \%$, OR: 3.8 [95\% CI: 1.7 to 8.5 ] for survival of discharge; 57.1 vs $16.9 \%$, OR: 6.6 [95\% CI: 2.9 to 14.9] for good CPC; and 100 vs $65.1 \%$ for good CPC among survival-to-discharge) compared with those 243 patients by DATCPR rescue. In 28 patients by bystander defibrillation rescue only one man without prehospital ROSC still achieved survival-to-discharge and good CPC.

Conclusion For OHCA patients at public locations, we found that a community-wide bystander defibrillation program were associated with excellent neurological outcome of CPC 1 and survival to hospital discharge that were significantly higher than those associated with DATCPR program.

Conflict of interest None

Funding None

\section{A WORLD RECORD FOR LIFE - A NATIONWIDE CPR INITIATIVE}

${ }^{1} \mathrm{~A}$ Mielke*, ${ }^{2} \mathrm{TW}$ Jensen, ${ }^{3} \mathrm{~N}$ Stark, ${ }^{4} \mathrm{P}$ Weber, ${ }^{5} \mathrm{G}$ Thomas, ${ }^{2} \mathrm{~F}$ Lippert, ${ }^{2} \mathrm{~F}$ Folke. ${ }^{1}$ Department of Cardiology, Gentofte University Hospital, Denmark; ${ }^{2}$ Emergency Medical Services Copenhagen, Denmark; ${ }^{3}$ Department of Anaesthesiology, Herlev University Hospital, Denmark; ${ }^{4}$ University of Copenhagen, Denmark; ${ }^{5}$ TrygFonden, Denmark

\subsection{6/10.1136/bmjopen-2018-EMS.84}

Aim Bystander cardiopulmonary resuscitation (CPR) is a cornerstone of improving survival in out-of-hospital cardiac arrests (OHCA). ${ }^{1}$ TrygFonden's World Record for Life aimed to show the public how easy it is to perform CPR by setting a world record in the number of people nationwide performing quality chest compression during 12 hours.

Method The record was set for 12 locations in Denmark over a 12 hour period on 22nd of May 2017. Each contribution was defined as $30 \mathrm{~s}$ of compression-only CPR on a Laerdal Q-CPR manikin with a Laerdal compression score of $\geq 65 \%$. $^{2}$ Compression score was based on compression depth, rate, conflict of interest, hands-off time, and placement of hands. Data was stratified by citizens or by attendees at the Emergency Medical Services Congress 2017 (EMS2017) in Copenhagen, and analysed using Wilcoxon rank test.

Results Out of 6094 participants, 5707 (94\%) reached a compression score $65 \%$ or more. Participants with a score of under $65 \%$ struggled with all components except compression rate compared to participants that reached 65\%. Comparing laypersons and EMS2017-attendees, both groups performed within guidelines. The world record reached 12 different TV broadcasts, 11 radio broadcasts, 30 printed newspapers, and 41 web newspapers from 1 st to 26th May 2017. The world record had approximately $3,739,000$ views on all platforms during the media coverage period.

Conclusion TrygFonden's World Record for Life showed an entire nation that untrained bystanders are able to provide effective CPR with 5707 reaching a compression score of $65 \%$ and $3,739,000$ media views.

\section{REFERENCES}

1. Rajan S, Wissenberg M, Folke F, Hansen SM, Gerds TA, Kragholm K, et al. Association of bystander cardiopulmonary resuscitation and survival according to ambulance response times after out-of-hospital cardiac arrest. Circulation 2016;134(25):2095-104.

2. http://cdn.laerdal.com/downloads-test/f3784/Att_2_to_00021778.pdf

\section{Conflict of interest None}

Funding The project was funded by TrygFonden.

\section{THE PRE-HOSPITAL MANAGEMENT OF ACUTE HEART} FAILURE: A CLINICAL AUDIT OF CURRENT PRACTICE

1J Price*, ${ }^{2} \mathrm{~B}$ Murphy-Jones, ${ }^{2} \mathrm{~T}$ Edwards. ${ }^{1}$ Cambridge University Hospitals NHS Trust, UK; ${ }^{2}$ London Ambulance Service, UK

\subsection{6/10.1136/bmjopen-2018-EMS.85}

Aim There has been a drive towards an increase in community-based management of heart failure. When patients experience acute heart failure (AHF), the complex nature of this condition poses diagnostic uncertainty for first responders. It is widely accepted that all patients should be transferred promptly to hospital, however with the introduction of prehospital diuresis, nitrate therapy and more recently non-invasive ventilation (NIV), the debate into the appropriateness and limitations of so-called 'stay-and-play' management strategies for patients in AHF has been re-ignited. We examine the current clinical assessment and management of AHF within the London Ambulance Service.

Method Ambulance Patient Report Forms (PRFs) from cases that were coded with heart failure, shortness of breath, cardiac problem and in cases of GTN administration. These cases were further analysed by a clinical review panel to identify patients with suspected AHF.

Results 182 patients were included in the analysis between April and November 2016. There was a 68\% compliance with national guidelines for clinical assessment (history, examination and ECG). 51 (28\%) patients presenting with AHF were appropriately identified and given a primary diagnosis of AHF by the attending clinician. $136(76 \%)$ patients in the analysis received sublingual nitrate therapy. 90 (49\%) patients received nitrates where there was no clinical indication. No patients in the analysis received NIV.

Conclusion Some aspects of AHF assessment and management are not consistent with national guidelines. Our work has further demonstrated the diagnostic challenges facing pre-hospital clinicians and the potential overuse of nitrate therapy in this patient group.

Conflict of interest None

Funding None

\section{HAEMODYNAMIC EFFECTS OF THE ENTERAL ADMINISTRATION OF TRANEXAMIC ACID IN AN EXPERIMENTAL MODEL OF HAEMORRHAGIC SHOCK}

G Babini*, D Olivari, D de Giorgio, L Staszewsky, R Latini, G Ristagno. IRCCS Istituto di Ricerche Farmacologiche Mario Negri, Milan, Italy

10.1136/10.1136/bmjopen-2018-EMS.86

Aim Systemic proteolysis has been proposed as part of the complex pathologic events occurring during haemorrhagic shock (HS). Hypoperfusion may increase permeability of the gut mucosa, promoting intestinal proteases translocation into the circulation and multiorgan failure ('autodigestion hypothesis'). ${ }^{1}$ The interruption of this cascade of events may improve systemic perfusion and organ functions.

Method The present study investigated the effects of the enteral administration of a protease inhibitor, i.e. tranexamic acid (TXA), on hemodynamics in a porcine model of controlled severe acute bleeding, fluid resuscitation and blood transfusion. Six animals underwent HS without any treatment while five animals were treated with enteral TXA. 